DOI: $10.6060 / \mathrm{mhc} 150351 \mathrm{~m}$

\title{
The Synthesis of Diamidediallylcalix[4]arene Derivatives as Ligand for Bromide Anion
}

\author{
Aliia S. Gusak, Polina E. Prokhorova, Yuri Yu. Morzherin ${ }^{\circledR}$ \\ Ural Federal University, 620002 Ekaterinburg, Russian Federation \\ ${ }^{\circledR}$ Corresponding authorE-mail: yu.yu.morzherin@urfu.ru
}

\begin{abstract}
Alkylation reaction of 5,17-diallylcalix[4]arene by the $\mathrm{N}$-alkyl and $\mathrm{N}$-aryl amides of chloroacetic acid was performed. NMR and UV titration of calix[4]arenes by bromide anion were carried out. It was shown that complex calixarene:bromide 2:1 was formed. The calixarene containing two $n$-butylamide moieties was determined to possess the most significant binding constant among all examined compounds.
\end{abstract}

Keywords: Calix[4]arene, bromide, UV titration, Job’s plot.

\section{Синтез диамидов диаллилкаликс[4]аренов как лигандов Аля аниона брома}

\author{
А. С. Гусак, П. Е. Прохорова, Ю. Ю. Моржерин ${ }^{\circledR}$ \\ Уральский федеральньй университет, 620002 Екатеринбург, Россия \\ ${ }^{\circledR}$ E-mail: yu.yu.morzherin@urfu.ru
}

\begin{abstract}
Была проведена реакиия алкилирования 5,17-диаллилкаликс[4]арена $N$-алкил и N-арил амидами хлоруксусной кислоты. Для определения констант связывания и состава комплекса полученных каликс[4]аренов с анионом брома были использованы методы ЯМР и УФ титрования. Было показано, что формируется комплексное соединение каликсарен:бромид 2:1. Выявлено, что каликсарен, содержащий два фрагмента н-бутиламида, обладает самой большой константой связывания среди всех исследованных соединений.
\end{abstract}

Ключевые слова: Каликс[4]арен, бромид, УФ титрование, график Джоба.

\section{Introduction}

In the last decade, considerable attention in supramolecular chemistry was paid to molecular recognition and self-assembled architectures. ${ }^{[1-4]}$ One of the main aspects of this multi-disciplinary field of chemistry is the design and creation of unusual compounds based on these receptors and sensors for biological and inorganic cation and anion..$^{[5-8]}$

It is known that compounds containing allyl group are good starting materials for polymerization reaction ${ }^{[9-12]}$ and chiral molecules receiving, ${ }^{[13-15]}$ but there are only few examples of such reactions in supramolecular chemistry. ${ }^{[16,17]}$ So, calixarene bearing allyl group is a promising molecule for design of various host compounds. To synthesize such ligands it is necessary to introduce specific moiety for anion/ cation recognition insight calix structure. For this purpose the amide group is widely used in supramolecular chemistry for receiving host molecules. ${ }^{[18-22]}$

Our research is devoted to the design of 5,17diallylcalix[4]arene derivatives containing two or four aryl(alkyl)amide moieties for study of their complex formation capacity to bind bromide anion. Concentration of this ion in nature water ranges from several microgram to several grams per liter. Anion $\mathrm{Br}$ may transform into oxidized forms $\mathrm{BrO}^{-}$and $\mathrm{BrO}_{3}^{-}$in the process of water disinfection by ozone and chlorine. Hypobromite and bromate react with organic ingredients of water resulting in bromoform, bromodichloromethane, bromophenols and other compounds. Organic and inorganic products of bromide transformations are carcinogenic and belong to the second class of danger. ${ }^{[23]}$ 


\section{Experimental}

${ }^{1} \mathrm{H}$ NMR spectra were recorded on a «Bruker» spectrometer (400 MHz for ${ }^{1} \mathrm{H}$ and $101 \mathrm{MHz}$ for ${ }^{13} \mathrm{C}$ ) in $\mathrm{CDCl}_{3}$, internal standard - TMS. Mass spectra were recorded on a mass spectrometer of MicrOTOF-Q II firm Bruker Daltonics series. UV spectra were obtained on a UV/VIS spectrometer «Lambda 35». Acetonitrile was dried over $\mathrm{P}_{2} \mathrm{O}_{5}$ and distillated. Amides of chloroacetic acid were received concerning literature. ${ }^{[2]}$ Other chemicals were used without any purification.

General method of 5,17-diallyl-25,27,26,28-hydroxy-calix[4] arene (1) alkylation. A mixture of calix[4]arene 1 (0.991 mmol) and potassium carbonate $(1.09 \mathrm{mmol})$ was refluxed in $25 \mathrm{ml}$ of acetonitrile for $1 \mathrm{~h}$. Then the amide of chloroacetic acid $(1.982 \mathrm{mmol})$ was added to the flask. The reaction mixture was refluxed for $24 \mathrm{~h}$. The solvent was evaporated, $\mathrm{CH}_{2} \mathrm{Cl}_{2}(50 \mathrm{ml})$ was added and washed with $1 \mathrm{~N} \mathrm{HCl}$ $(50 \mathrm{ml} \times 3)$. The organic layer was dried $\left(\mathrm{MgSO}_{4}\right)$ and evaporated. Crude product was crystallized from ethanol and purified by column chromatography (eluent petroleum ether:ethyl acetate 1:1).

5,17-Diallyl-25,27-di(N-phenylcarbamoylmethoxy)-26,28hydroxycalix [4] arene (2). Red powder, yield $229 \mathrm{mg}$ (30\%). Found, $\%$ : C, 77.80; H, 5.99; N, 3.50. $\mathrm{C}_{50} \mathrm{H}_{48} \mathrm{O}_{6} \mathrm{~N}_{2}$. Calculated, \%: C, 77.90; $\mathrm{H}, 6.01 ; \mathrm{N}, 3.63$. Mass-spectra (ESI) $\mathrm{m} / z: 793\left[(\mathrm{M}+\mathrm{Na})^{+}\right]$. ${ }^{1} \mathrm{H}$ NMR $\left(\mathrm{CDCl}_{3}, 298 \mathrm{~K}\right) \delta_{\mathrm{H}}$ ppm: $10.20(2 \mathrm{H}, \mathrm{s}, \mathrm{NH}) ; 7.37\left(4 \mathrm{H}, \mathrm{d} J^{3}=8.0 \mathrm{~Hz}\right.$, $\mathrm{ArH}), 7.21\left(4 \mathrm{H}, \mathrm{t} J^{3}=8.0 \mathrm{~Hz}, \mathrm{ArH}\right), 7.14\left(2 \mathrm{H}, \mathrm{t} J^{3}=8.0 \mathrm{~Hz}, \mathrm{ArH}\right)$, $7.04\left(4 \mathrm{H}, \mathrm{d} J^{3}=7.6 \mathrm{~Hz}, \mathrm{ArH}\right), 6.85(4 \mathrm{H}, \mathrm{s}, \mathrm{ArH}) ; 6.75\left(2 \mathrm{H}, \mathrm{t} J^{3}=\right.$ 7.6 Hz, ArH $) ; 5.98-5.78\left(2 \mathrm{H}, \mathrm{m}, \mathrm{CH}_{2}-\mathrm{CH}=\mathrm{CH}_{2}\right) ; 5.10-4.97(4 \mathrm{H}, \mathrm{m}$, $\left.\mathrm{CH}_{2}-\mathrm{CH}=\mathrm{CH}_{2}\right) ; 4.6\left(4 \mathrm{H}, \mathrm{s}, \mathrm{OCH}_{2} \mathrm{CO}\right) ; 4.24\left(4 \mathrm{H}, \mathrm{d} J^{2}=12.6 \mathrm{~Hz}\right.$, $\left.\mathrm{ArCH}_{2} \mathrm{Ar}\right) ; 3.54\left(4 \mathrm{H}, \mathrm{d} J^{2}=12.6 \mathrm{~Hz}, \mathrm{ArCH}_{2} \mathrm{Ar}\right) ; 3.17\left(4 \mathrm{H}, \mathrm{d} J^{3}=6.4\right.$, $\left.\mathrm{CH}_{2}-\mathrm{CH}=\mathrm{CH}_{2}\right) .{ }^{13} \mathrm{C} \mathrm{NMR}\left(\mathrm{CDCl}_{3}, 298 \mathrm{~K}\right) \delta_{\mathrm{C}} \mathrm{ppm}: 163.54,155.68$, $149.12,138.34,138.01,136.77,132.82,132.60,130.62,129.92$, 129.21, 127.55, 124.67, 120.99, 115.77, 55.55, 39.54, 31.95.

5,17-Diallyl-25,27-di(N-p-methoxyphenylcarbamoylmethoxy)-26,28-hydroxycalix [4]arene (3). Brown powder, yield $247 \mathrm{mg}$ (30\%). Found, \%: C, 75.56; H, 5.78; N, 3.50. $\mathrm{C}_{52} \mathrm{H}_{52} \mathrm{O}_{8} \mathrm{~N}_{2}$. Calculated, \%: C, 75.16; H, 6.06; N, 3.37. Mass-spectra (ESI) $\mathrm{m} / \mathrm{z}$ : $853\left[(\mathrm{M}+\mathrm{Na})^{+}\right] .{ }^{1} \mathrm{H}$ NMR $\left(\mathrm{CDCl}_{3}, 298 \mathrm{~K}\right) \delta_{\mathrm{H}} \mathrm{ppm}: 10.19(2 \mathrm{H}, \mathrm{s}$, $\mathrm{NH}) ; 8.41(2 \mathrm{H}, \mathrm{s}, \mathrm{OH}) ; 7.33\left(4 \mathrm{H}, \mathrm{d} J^{3}=9.0 \mathrm{~Hz}, \mathrm{ArH}\right) ; 7.13(4 \mathrm{H}, \mathrm{d}$ $\left.J^{3}=7.5 \mathrm{~Hz}, \mathrm{ArH}\right) ; 6.86(4 \mathrm{H}, \mathrm{s}, \mathrm{ArH}) ; 6.76\left(2 \mathrm{H}, \mathrm{t} J^{3}=7.5 \mathrm{~Hz}, \mathrm{ArH}\right)$; $6.75\left(4 \mathrm{H}, \mathrm{d} J^{3}=9.0 \mathrm{~Hz}, \mathrm{ArH}\right) ; 5.87-5.77\left(2 \mathrm{H}, \mathrm{m}, \mathrm{CH}_{2}-\mathrm{CH}=\mathrm{CH}_{2}\right)$; 5.10-4.96 (4H, m, $\left.\mathrm{CH}_{2}-\mathrm{CH}=\mathrm{CH}_{2}\right) ; 4.60\left(4 \mathrm{H}, \mathrm{s}, \mathrm{OCH}_{2} \mathrm{CO}\right) ; 4.22(4 \mathrm{H}$, d $\left.J^{2}=13.2 \mathrm{~Hz}, \mathrm{ArCH}_{2} \mathrm{Ar}\right) ; 3.80\left(6 \mathrm{H}, \mathrm{s}, \mathrm{OCH}_{3}\right) ; 3.55\left(4 \mathrm{H}, \mathrm{d} J^{2}=13.2\right.$ $\left.\mathrm{Hz}, \mathrm{ArCH}_{2} \mathrm{Ar}\right) ; 3.13\left(4 \mathrm{H}, \mathrm{d} J^{3}=6.8 \mathrm{~Hz}, \mathrm{CH}_{2}-\mathrm{CH}=\mathrm{CH}_{2}\right) .{ }^{13} \mathrm{C} \mathrm{NMR}$ $\left(\mathrm{CDCl}_{3}, 298 \mathrm{~K}\right) \delta_{\mathrm{C}} \mathrm{ppm}: 164.74,156.48,149.12,138.34,138.01$, $136.77,132.82,132.60,130.62,129.92,129.21,127.55,120.99$, $115.77,55.55,39.54,39.46,31.95$.

5,17-Diallyl-25,27-di(N-p-methylphenylcarbamoylmethoxy)26,28-hydroxycalix[4]arene (4). Violet powder, yield $277 \mathrm{mg}$ (35\%). Found, \%: C, 77.80; H, 6.25; N, 3.34. $\mathrm{C}_{52} \mathrm{H}_{52} \mathrm{O}_{6} \mathrm{~N}_{2}$. Calculated, \%: C, 78.17; H, 6.31; N, 3.51. Mass-spectra (ESI) $m / z: 799$ $\left[(\mathrm{M}+\mathrm{H})^{+}\right] .{ }^{1} \mathrm{H}$ NMR $\left(\mathrm{CDCl}_{3}, 298 \mathrm{~K}\right) \delta_{\mathrm{H}} \mathrm{ppm}: 10.18(2 \mathrm{H}, \mathrm{s}, \mathrm{NH})$; $8.16(2 \mathrm{H}, \mathrm{s}, \mathrm{OH}) ; 7.43\left(4 \mathrm{H}, \mathrm{d} J^{3}=8.2 \mathrm{~Hz}, \mathrm{ArH}\right) ; 7.17\left(4 \mathrm{H}, \mathrm{d} J^{3}=\right.$ $8.2 \mathrm{~Hz}, \mathrm{ArH}) ; 7.04$ (4H, d $\left.J^{3}=7.4 \mathrm{~Hz}, \mathrm{ArH}\right) ; 6.85$ (4H, s, ArH); 6.73 $\left(2 \mathrm{H}, \mathrm{t} J^{3}=7.4 \mathrm{~Hz}, \mathrm{ArH}\right) ; 5.93-5.79\left(2 \mathrm{H}, \mathrm{m}, \mathrm{CH}_{2}-\mathrm{CH}=\mathrm{CH}_{2}\right) ; 5.06-$ $5.01\left(4 \mathrm{H}, \mathrm{m}, \mathrm{CH}_{2}-\mathrm{CH}=\mathrm{CH}_{2}\right) ; 4.23\left(4 \mathrm{H}, \mathrm{d} J^{2}=13.2 \mathrm{~Hz}, \mathrm{ArCH}_{2} \mathrm{Ar}\right)$; $4.19\left(4 \mathrm{H}, \mathrm{s}, \mathrm{OCH}_{2} \mathrm{CO}\right) ; 3.52\left(4 \mathrm{H}, \mathrm{d} J^{2}=13.2 \mathrm{~Hz}, \mathrm{ArCH}_{2} \mathrm{Ar}\right) ; 3.17$ $\left(4 \mathrm{H}, \mathrm{d} \mathrm{J}^{3}=6.4, \mathrm{CH}_{2}-\mathrm{CH}=\mathrm{CH}_{2}\right) ; 2.33\left(6 \mathrm{H}, \mathrm{s}, \mathrm{ArCH}_{3}\right) .{ }^{13} \mathrm{C} \mathrm{NMR}$ $\left(\mathrm{CDCl}_{3}, 298 \mathrm{~K}\right) \delta_{\mathrm{C}} \mathrm{ppm}: 163.70,155,86,148.89,147.02,137.53$, $134.98,134.18,133.58,129.63,128.98,128.30,128.18,122.16$, $115.65,42.89,39.37,31.78,31.21$.

5,17-Diallyl-25,27-di(N-butylcarbamoylmethoxy)-26,28hydroxycalix [4]arene (5). Yellow powder, yield $390 \mathrm{mg}$ (54\%). Found, \%: C, 76.23; $\mathrm{H}, 7.37 ; \mathrm{N}, 3.87 . \mathrm{C}_{46} \mathrm{H}_{54} \mathrm{O}_{6} \mathrm{~N}_{2}$. Calculated, \%: C, 75.59; H, 7.45; N, 3.83. Mass-spectra (ESI) $m / z$ : $753\left[(\mathrm{M}+\mathrm{Na})^{+}\right]$. ${ }^{1} \mathrm{H}$ NMR $\left(\mathrm{CDCl}_{3}, 298 \mathrm{~K}\right) \delta_{\mathrm{H}} \mathrm{ppm}: 7.75(2 \mathrm{H}, \mathrm{s}, \mathrm{NH}) ; 7.03(4 \mathrm{H}$, d $\left.J^{3}=7.4 \mathrm{~Hz}, \mathrm{ArH}\right) ; 6.84(4 \mathrm{H}, \mathrm{s}, \operatorname{ArH}) ; 6.72\left(2 \mathrm{H}, \mathrm{t} J^{3}=7.4 \mathrm{~Hz}\right.$, $\mathrm{ArH})$; 5.98-5.82 (2H, m, $\left.\mathrm{CH}_{2}-\mathrm{CH}=\mathrm{CH}_{2}\right) ; 5.19-5.01(4 \mathrm{H}, \mathrm{m}$,
$\left.\mathrm{CH}_{2}-\mathrm{CH}=\mathrm{CH}_{2}\right) ; 4.41\left(4 \mathrm{H}, \mathrm{d} J^{2}=14.0 \mathrm{~Hz}, \mathrm{ArCH}_{2} \mathrm{Ar}\right) ; 4.18(4 \mathrm{H}, \mathrm{s}$, $\left.\mathrm{OCH}_{2} \mathrm{CO}\right) ; 3.93\left(4 \mathrm{H}, \mathrm{d} J^{2}=14.0 \mathrm{~Hz}, \mathrm{ArCH}_{2} \mathrm{Ar}\right) ; 3.39-3.25(4 \mathrm{H}, \mathrm{m}$, $\left.\mathrm{NH}-\mathrm{CH}_{2}-\mathrm{CH}_{2}-\mathrm{CH}_{2}-\mathrm{CH}_{3}\right) ; 3.26\left(4 \mathrm{H}, \mathrm{d} J^{3}=6.8 \mathrm{~Hz}, \mathrm{CH}_{2}-\mathrm{CH}=\mathrm{CH}_{2}\right)$; 1.69-1.59 (4H, m, NH-CH $\left.-\mathrm{CH}_{2}-\mathrm{CH}_{2}-\mathrm{CH}_{3}\right) ; 1.50-1.42(4 \mathrm{H}, \mathrm{m}$, $\left.\mathrm{NH}-\mathrm{CH}_{2}-\mathrm{CH}_{2}-\mathrm{CH}_{2}-\mathrm{CH}_{3}\right) ; 1.07-0.94\left(6 \mathrm{H}, \mathrm{t} J^{3}=7.2 \mathrm{~Hz}, \mathrm{NH}-\mathrm{CH}_{2}-\right.$ $\left.\mathrm{CH}_{2}-\mathrm{CH}_{2}-\mathrm{CH}_{3}\right) \cdot{ }^{13} \mathrm{C} \mathrm{NMR}\left(\mathrm{CDCl}_{3}, 298 \mathrm{~K}\right) \delta_{\mathrm{C}} \mathrm{ppm}: 169.13,168.52$, $147.56,145.39,137.93,134.60,129.51,128.29,123.45,115.64$, 74.70, 74.41, 41.61, 41.39, 39.65, 31.23, 22.57, 22.52 .

5,17-Diallyl-25,26,27,28-tert( $N$ - isopropylcarbamoylmethoxy)calix [4] arene (6). Orange powder, yield $446 \mathrm{mg}$ (50\%). Found, \%: C, 75.53; H, 7.76; N, 3.68. $\mathrm{C}_{44} \mathrm{H}_{50} \mathrm{O}_{6} \mathrm{~N}_{2}$. Calculated, \%: C, 75.19; $\mathrm{H}, 7.17$; N, 3.99. Mass-spectra (ESI) $\mathrm{m} / z$ : $901\left[(\mathrm{M}+\mathrm{H})^{+}\right]$. ${ }^{1} \mathrm{H}$ NMR $\left(\mathrm{CDCl}_{3}, 298 \mathrm{~K}\right) \delta_{\mathrm{H}}$ ppm: $7.69(4 \mathrm{H}, \mathrm{s}, \mathrm{NH}) ; 6.75(4 \mathrm{H}, \mathrm{s}, \mathrm{ArH}) ; 6.41$ $\left(2 \mathrm{H}, \mathrm{t} J^{3}=7.0 \mathrm{~Hz}, \mathrm{ArH}\right) ; 6.25\left(4 \mathrm{H}, \mathrm{d} J^{3}=7.0 \mathrm{~Hz}, \mathrm{ArH}\right) ; 5.98-5.88$ $\left(2 \mathrm{H}, \mathrm{m}, \mathrm{CH}_{2}-\mathrm{CH}=\mathrm{CH}_{2}\right) ; 5.07-5.02\left(4 \mathrm{H}, \mathrm{m}, \mathrm{CH}_{2}-\mathrm{CH}=\mathrm{CH}_{2}\right) ; 4.50$ $\left(4 \mathrm{H}, \mathrm{s}, \mathrm{OCH}_{2} \mathrm{CO}\right) ; 4.43\left(4 \mathrm{H}, \mathrm{d} J^{2}=13.6 \mathrm{~Hz}, \mathrm{ArCH}_{2} \mathrm{Ar}\right) ; 4.29(4 \mathrm{H}$, $\left.\mathrm{s}, \mathrm{OCH}_{2} \mathrm{CO}\right) ; 4.17-4.08\left(4 \mathrm{H}, \mathrm{m}, \mathrm{CH}\left(\mathrm{CH}_{3}\right)_{2}\right) ; 3.25\left(4 \mathrm{H}, \mathrm{d} J^{3}=6.8\right.$, $\left.\mathrm{CH}_{2}-\mathrm{CH}=\mathrm{CH}_{2}\right) ; 3.18\left(4 \mathrm{H}, \mathrm{d} J^{2}=13.6 \mathrm{~Hz}, \mathrm{ArCH}_{2} \mathrm{Ar}\right) ; 1.27(12 \mathrm{H}, \mathrm{d}$ $\left.J^{3}=6.8, \mathrm{CH}_{3}\right) ; 1.14\left(12 \mathrm{H}, \mathrm{d} J^{3}=6.8, \mathrm{CH}_{3}\right) \cdot{ }^{13} \mathrm{C} \mathrm{NMR}\left(\mathrm{CDCl}_{3}, 298 \mathrm{~K}\right)$ $\delta_{\mathrm{C}}$ ppm: $169.13,168.52,147.56,145.39,137.93,134.60,129.51$, $128.29,123.45,115.64,74.70,74.41,41.61,41.39,39.65,31.23$, $22.57,22.52$.

\section{Results and Discussion}

\section{Synthesis}

Starting material for our research was calix[4]arene $\mathbf{1}\left[{ }^{[25]}\right.$ with two allyl groups at the upper rim. Cases for tetrabut not di-O-alkylation of compound $\mathbf{1}$ are described. ${ }^{[26,27]} \mathrm{We}$ assumed that $O$-alkylation of 1,3-dicalixarene 1 with two allyl groups at the upper rim can proceed at 1,3-positions. Firstly, we synthesized calix[4]arene 1 using known procedure. ${ }^{[25]}$ Then we carried out the alkylation of compound 1 with 2 equivalents of the chloroacetic acid amides in the presence of potassium carbonate as the base in dry acetonitrile as solvent to obtain the products of di-substitution 2-5 (Scheme 1).

The structures of all molecules were confirmed by ${ }^{1} \mathrm{H}$, ${ }^{13} \mathrm{C}$ NMR and mass-spectrometry. According to these data two molecules of alkylating agent were added. Aromatic protons of calix[4]arene were characterized as singlet, doublet and triplet. Three signals of allyl group were observed as intrinsic multiplet at approximately $5.8-6.0 \mathrm{ppm}, 5.0 \mathrm{ppm}$ and doublet at 3.17-3.26 ppm. Two doublets with coupling constant characteristic for geminal interaction were referred to bridging protons. For example, ${ }^{1} \mathrm{H}$ NMR spectrum of compound 3 consists of signals for aromatic protons of calixarenes triplet at $6.76 \mathrm{ppm}$, doublet at $7.13 \mathrm{ppm}$ with coupling constant $7.5 \mathrm{~Hz}$, and singlet at $6.86 \mathrm{ppm}$ as well. At the same time aromatic protons of alkylating agent appear as two doublets at 7.33 and $6.75 \mathrm{ppm}$ with $J^{3}=9.0 \mathrm{~Hz}$, where in signals for calixarenes triplet and alkylation agent duplet are over lapped. The protons of allyl groups give two multiplets at 5.87-5.77 and 5.10-4.96 ppm as well as doublet at 3.13 ppm with coupling constant $6.8 \mathrm{~Hz}$. Characteristic doublets for equatorial and axial bridging protons are observed at 4.22 and $3.55 \mathrm{ppm}$ with $J^{2}=13.2 \mathrm{~Hz}$. $\mathrm{OCH}_{2} \mathrm{CO}$ protons appear at $4.6 \mathrm{ppm}$ as a singlet.

The alkylation of calix[4]arene $\mathbf{1}$ by alkylamide of chloroacetic acid gives unexpected results. We have received products of di-substitution using butylamide of chloro-acetic acid $\mathbf{5}$ and tetra-substitution using isopropylamide of chloro- 


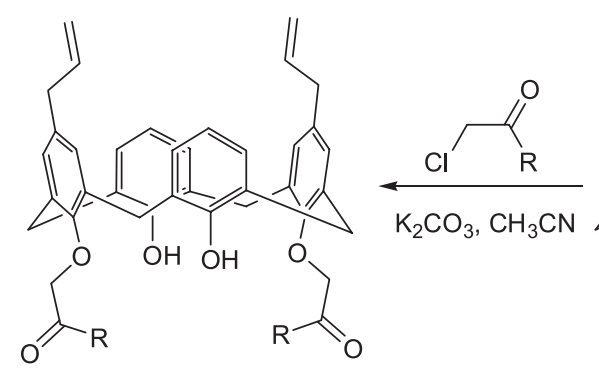

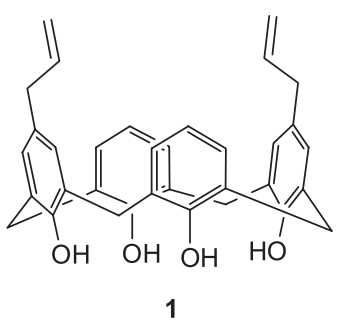

$\mathrm{R}=$<smiles>CCCCN</smiles>

Scheme 1. Synthesis of calix[4]arenes 2-6.

acetic acid 6. In ${ }^{1} \mathrm{H}$ NMR of calixarene 6 two set of alkylating agent signals were observed.

The reaction capacity of alkyl containing agents for alkylation reaction is greater than for aromatic ones.

\section{Titration Studies}

The NMR titration is widely used for studying calixarene complex formation by analyzing of chemical shifts changing. ${ }^{[28-30]}$ So the ability of the synthesized calixarenes to form complex with bromide as anionic guest was determined by known method, ${ }^{[31]}$ using compound $\mathbf{2}$ as reference molecule (Figure 1a).

Tetrabutylammonium bromide (TBAB) solution $(0.5 \mathrm{mM})$ was added gradually from 0 to 10 equivalents to the calixarene 2 solution $(0.5 \mathrm{mM})$ in $\mathrm{CDCl}_{3}$. Addition of bromide anion has induced the upfield shift for phenolic protons from 8.50 and $8.29 \mathrm{ppm}$ to 8.41 and $8.21 \mathrm{ppm}$, the same tendency was remarked for NH protons (Figure 1a). It indicates that complexation of $\mathrm{Br}^{-}$by calix[4]arene 2 takes place via hydrogen-bonding interactions with amide and phenolic proton moieties.

A Job plot for 2 and $\mathrm{Br}^{-}$has shown a maximum at 0.25 mole fraction, indicating a 4:1 amide moiety/bromide binding stoichiometry (Figure 1b). Due to structure of calixarene $\mathbf{2}$ has two amide groups, we suggested that dimerized tunnel-shaped structure $\mathbf{8}$ was formed in this interaction (Figure 2).

Rose-Drago method for UV-visible spectroscopy was used to calculate binding constants of TBAB $\mathbf{2}-\mathbf{6}$ by calix[4] arene. The experiments were carried out in $\mathrm{CH}_{3} \mathrm{CN}$ and $\mathrm{CHCl}_{3}$ at room temperature (Table 1) using known methodology. Typically, the solutions of TBAB $(0.5 \mathrm{mM})$ and calixarene $(0.5 \mathrm{mM})$ were mixed in several different ratios (from 10:0 to $0: 10$ equivalent ratio for calixarene:TBAB). Absorption spectra were measured using a Lambda spectrophotometer in the range of 200-600 $\mathrm{nm}$. Job's plot for each calixarene showed 2:1 calixarene:TBAB stoichiometry as well as in NMR titration experiment. Binding constant values for all compounds 2-6 are shown in the Table 1.
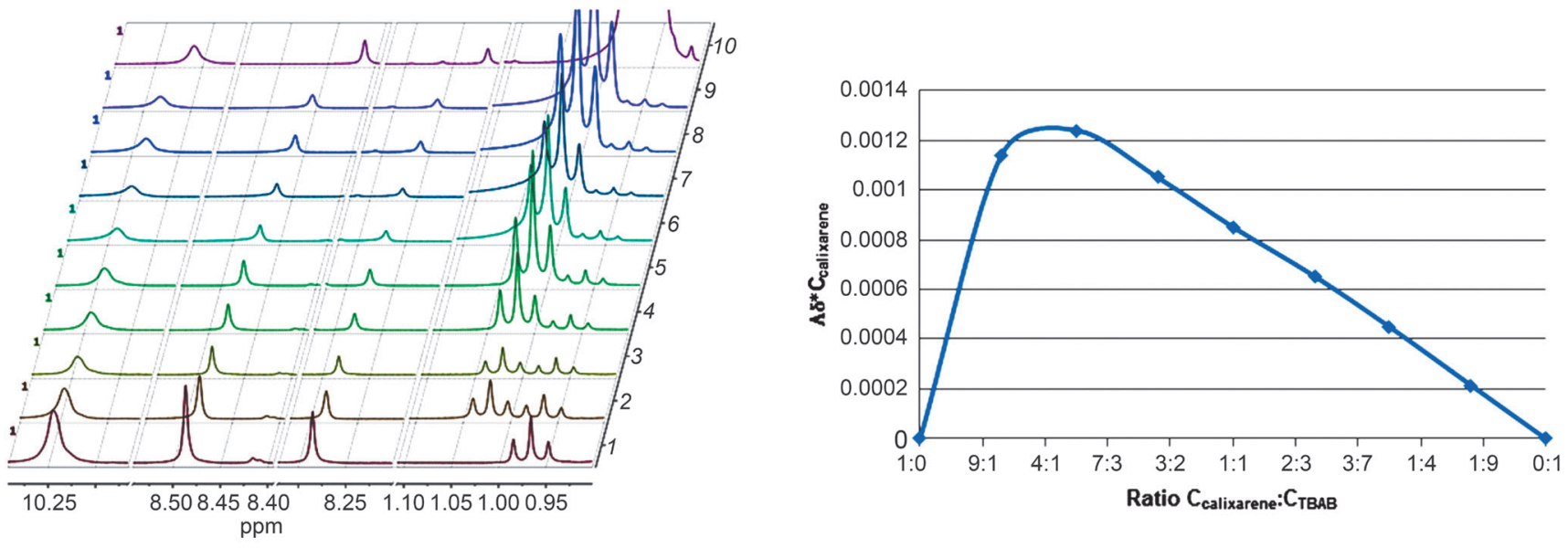

Figure 1. a) Comparing of NMR spectra at titration; b) Jobs plot for NMR titration. 


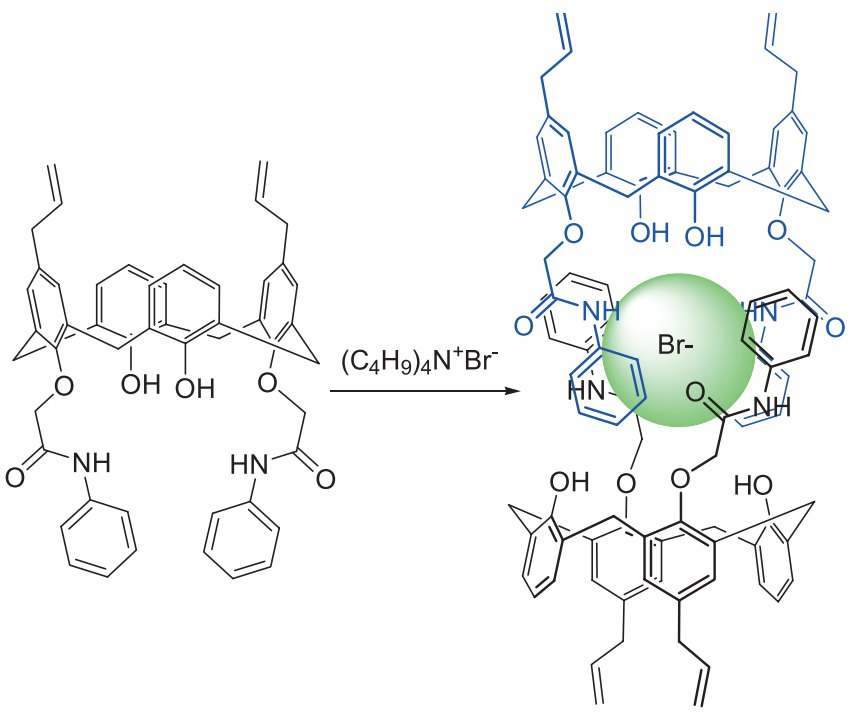

Figure 2. Estimated complex structure.

Table 1.

\begin{tabular}{cc}
\hline Calix [4]arene & Binding constant, $\mathrm{l}^{2} \cdot \mathrm{mol}^{-2}$ \\
\hline $\mathbf{2}$ & 1210 \\
$\mathbf{3}$ & 1430 \\
$\mathbf{4}$ & 1360 \\
$\mathbf{5}$ & 17600 \\
$\mathbf{6}$ & 13900 \\
\hline
\end{tabular}

These data show that calix[4] arene 5 with $n$-butyl amide moieties is the most suitable complexation agent. While binding ability of compounds $\mathbf{2} \mathbf{- 4}$ with aromatic fragments is approximately ten times worse then that of calixarenes $\mathbf{5}$ and 6. It may be explained by the nature of TBAB salt: it contains aliphatic butyl fragments, chemically similar to $n$-butyl and $i$-propyl groups.

\section{Conclusions}

Our group has reported the method for synthesis of new chiral calix[4]arenes containing amide moieties. The use of aromatic amides of chloroacetic acid as the alkylation agent gives 5,17-diallyl-25,27-disubstituted calix[4]arenes. In contrast, the alkylation by alkyl amides of chloroacetic acid is less selective and could result in di- and tetra-substituted calix[4]arenes.

The titration study has shown that calix[4]arene 2 forms a complex with the bromide anion in 2:1 ratio. Also the binding constants for the all synthesized calix[4]arenes were calculated.

Acknowledgements. The work was supported by Act 211 Government of the Russian Federation, contract № 02. A03.21.0006, State task of Ministry of Education and Science No 4.560.2014/K.

\section{References}

1. Pasquale S., Sattin S., Escudero-Adan E.C., MartinezBelmonte M., de Mendoza J. Nature Commun. 2012, 3 1-7.

2. Dasary H., Jagan R., Chand D.K. Chem. Eur. J. 2015, 21, 1499-1507.

3. Aydogan A., Lee G., Lee C.-H., Sessler J.L. Chem. Eur. J. 2015, 21, 2368-2376.

4. Zheng G., Chen M., Liu X., Zhou J., Xie J., Diao G. Electrochimica Acta 2014, 136, 301-309.

5. Chawla H.M., Kar J.R., Siddiqui W.A., Kumar N., Black D.S. Tetrahedron Lett. 2014, 55, 4517-4520.

6. Chawla H.M., Goel P., Munjal P. Tetrahedron Lett. 2015, 56, 682-685.

7. Mummidivarapua V.V.S., Pathaka R.K., Hingeb V.K., Dessingoua J., Rao C.P. Supramol. Chem. 2014, 26, 538-546.

8. Mutihac L., Enache I.V., Diacu E., Mutihac R.-C., Vicens J. Supramol. Chem. 2014, 26, 521-525.

9. He X., Fan J., Zhang F., Li R., Pollack K.A., Raymond J.E., Zou J., Wooley K.L. J. Mater. Chem. B 2014, 2, 8123-8130.

10. Haupt S., Handke M., Kuhnert R., Poetsch M., Kersting B. Tetrahedron 2014, 70, 5254-5259.

11. Liu Y., Hao Z., Lv S., Huang J., Liao C., Run M. Polymer 2015, 57, 29-38.

12. Gusak A.S., Ivanova E.A., Prokhorova P.E., Rusinov G.L., Verbitskiy E.V., Morzherin Yu.Yu. Russ. Chem. Bull. 2014, 63, 1395-1398.

13. Gładkowski W., Skrobiszewski A., Mazur M., Siepka M., Białońska A. Eur. J. Org. Chem. 2015, 605-615.

14. Deng W.-H., Ye F., Bai X.-F., Zheng Z.-J., Cui Y.-M., Xu L.-W. ChemCatChem 2015, 7, 75-79.

15. Rapi Z., Bakó P., Drahos L., Keglevich G. Heteroatom Chem. 2015, 26, 63-71.

16. Haupt S., Handke M., Kuhnert R., Poetsch M., Kersting B. Tetrahedron 2014, 70, 5254-5259.

17. Pitarch M., McKee V., Nieuwenhuyzen M., McKervey M.A. J. Org. Chem. 1998, 63, 946-951.

18. Ozkan S.C., Yilmaz A., Arslan E., Acik L., Sayin U., Mutlu E.G. Supramol. Chem. 2015, 27, 255-267.

19. Ansari S.A., Mohapatraa P.K., Iqbal M.I., Huskens J., Verboom W. J. Chromatography A 2014, 1334, 79-86.

20. Hung H.-C., Chang Y.-Y., Luo L., Hung C.-H., Diau E.W.-G., Chung W.-S. Photochem. Photobiol. Sci. 2014, 13, 370-379.

21. Cafeo G., Gattuso G., Kohnke F.H., Papanikolaou G., Profumo A., Rosano C. Chem. Eur. J. 2014, 20, 1658-1668.

22. Kulyashova A.E., Geide I.V., Prokhorova P.E., Morzherin Yu.Yu. Russ. J. Org. Chem. 2014, 50, 565-570.

23. Methodical instruction 4.1.2587-10 Spectrophotometrical determination of bromide ions in the drinking water (in Russ.)

24. Zhaowen L., Li Z., Chunfen X., Yong Y., Fanbo Z., Kaixun H. Med. Chem. Res. 2007, 16, 380-391.

25. van Loon J.-D., Arduini A., Coppi L., Verboom W., Pochini A., Ungaro R., Harkema S., Reinhoudt D.N. J. Org. Chem. 1990, $55,5639-5646$.

26. Dondoni A., Kleban M., Hu X., Marra A., Banks H.D. J. Org. Chem. 2002, 67, 4722-4733.

27. Pitarch M., McKee V., Nieuwenhuyzen M., McKervey M.A. J. Org. Chem. 1998, 63, 946-951.

28. Hu X.-Y., Peng S., Guo D.-S., Ding F., Liu Y. Supramol. Chem. 2015, 27, 336-345.

29. Mendez-Arroyo J., Barroso-Flores J., Lifschitz A.M., Sarjeant A.A., Stern C.L., Mirkin C.A. J. Am. Chem. Soc. 2014, 136, 10340-10348.

30. Gaeta C., Talotta C., Sala P.D., Margarucci L., Casapullo A., Neri P. J. Org. Chem. 2014, 79, 3704-3708.

31. Hirose K. Determination of Binding Constants. In: Analytical Methods in Supramolecular Chemistry, Ch. 2 (Schalley C., Ed.), Weinheim, Germany: Wiley-VCH, 2006. pp. 17-54. 